CALT-68-1921

DOE RESEARCH AND

DEVELOPMENT REPORT

\title{
Scattering from Electroweak Strings ${ }^{\star}$
}

\author{
Hoi-Kwong Lo $^{\dagger}$ \\ California Institute of Technology, Pasadena, CA 91125
}

\begin{abstract}
The scattering of a charged fermion from an electroweak string is studied. Owing to an amplification of the wave function at the core radius, the cross sections for helicity flip processes can be largely enhanced. For $0<\sin ^{2} \theta_{w}<1 / 2$ (where $\theta_{w}$ is the Weinberg angle), $\omega \sim k \sim m_{e}$ and $k R \ll 1$, we show that the helicity flip differential cross section for electrons is of the order $m_{e}^{-1}$ and is independent of angle. We compare our results with those obtained in calculations of rates for baryon number violating processes in the core of a cosmic string. In that case, while the enhancement is a general phenomenon, its actual magnitude is extremely sensitive to the fractional flux carried by the string core. Apart from showing the existence of a similar enhancement effect for non-topological strings, our results indicate that in some models the magnitude of enhancement can be rendered much less sensitive to the value of the parameters in the theories. Scattering of particles off semi-local strings and axion strings are also considered.
\end{abstract}

* This work supported in part by the U.S. Department of Energy under Grant No. DE-FG0392-ER40701

$\dagger$ HKL@THEORY.CALTECH.EDU

$\ddagger$ address after 1 September: Institute for Advanced Study, Princeton, NJ 08540 


\section{Introduction}

Some years ago, Callan ${ }^{[1]}$ and Rubakov $^{[2]}$ (see also Wilczek ${ }^{[3]}$ ) showed that a grand unified monopole may catalyze baryon number violating processes with strong interaction cross sections rather than the much smaller geometric cross sections. This enhancement effect can be understood as a consequence of a large amplification of the fermionic wave functions near the location of the monopole. ${ }^{[4]}$

A similar enhancement of cross section also occurs for cosmic strings with frac-

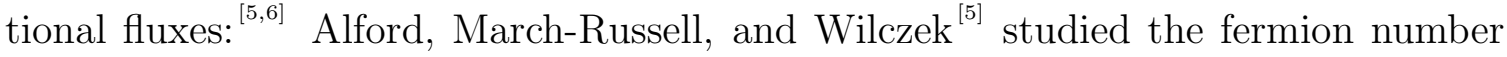
violating process in a cosmic string core due to a Yukawa coupling. In their model, there are two fermions with equal $U(1)$ charges and two scalar fields. The first scalar field, $\eta$, acts as the Higgs field and thus condenses outside string core. The second scalar field, $\phi$, which has a Yukawa coupling to the two fermion fields, condenses within the core. In the limit $k R \ll \nu R \ll 1$, where $k$ is the momentum of the incident fermion, $R$ is the size of the core and $\nu=\lambda\langle\phi\rangle(\lambda$ being the Yukawa coupling constant), they found generic enhancement by large factors over the naive, geometric cross section. Maximal enhancement occurs when $\frac{d \sigma}{d \theta} \sim \frac{1}{k}$.

A prominent feature of their results is that while a large enhancement of the fermion number violating process is a general phenomenon, its actual magnitude is extremely sensitive to the $U(1)$ charge of the fermions. For instance, changing the charge from $\alpha=1 / 2$ to $\alpha=1 / 4$ results in a diminuation of the inelastic cross section by around 15 orders of magnitude. By assigning baryon numbers to the fermions and scalars, their results imply that the exact magnitude of the baryon number violating process is very sensitive to the details of the grand unification model under consideration. Since there are uncertainties in our experimental determination of low energy parameters such as the Weinberg angle, it might be hard for us to say for sure whether a model is phenomenologically feasible. The point is that a slight error made in our determination of the values of such parameters leads to a huge variation in the rate of baryogenesis and may render a feasible model unfeasible and vice versa. For this reason, we would like to ask the following question: Is this sensitivity a 
generic feature or is it model-dependent? In other words, can we construct a model where the inelastic cross section is less sensitive to the values of the parameters?

A hint to the answer to this question comes from the investigation made by Perkins et $a l^{[6]}$ In their paper, the cross section for a baryon number violating process was derived using first order perturbation theory in quantum field theories. The transition matrix element between an initial state $|\psi\rangle$ and a final state $\left|\psi^{\prime}\right\rangle$ is given by $\mathcal{A}=\left\langle\psi^{\prime}\left|\int d^{4} x \mathcal{L}_{I}(x)\right| \psi\right\rangle$. The computation was divided into two steps. Firstly, they evaluated $\mathcal{A}$ using free fermion spinors, resulting in the "geometric" cross section. In the second step, they solved the Dirac equation with the appropriate boundary conditions to determine the amplitude of the spinor at the core radius $R$ and defined the amplification factor $A$ as the ratio of the amplitude of the actual spinor to that of a free spinor. Since the cross section is proportional to $\mathcal{A}^{2}$ and since $\mathcal{A}$ involves two spinors, the catalysis cross section is enhanced by a factor $A^{4}$ over the geometric cross section. This argument relies on the fact that the amplification factor for the initial state is the same as that for the final state. It might be possible for us to construct models with different amplification factors for the initial and final states. If the two amplifications have opposite dependence on the parameters, the overall amplification, which is the product of the two amplification factors, will then be insensitive to the parameters in the model.

In this paper, we study the scattering of a charged fermion from an electroweak string. We show that for $0<\sin ^{2} \theta_{w}<1 / 2\left(\theta_{w}=\right.$ Weinberg angle), $\omega \sim k \sim m_{e}$ and $k R \ll 1$, the helicity flip differential cross section for electrons is of the order $m_{e}^{-1}$. A delicate cancellation of the dependence of the two amplification factors on the Weinberg angle indeed occurs within this regimé. We would like to remark that the differential cross section in this regimé is dominated by a single partial wave and is thus independent of angle.

Incidentially, our results illustrate that the analysis of the enhancement effects for cosmic strings can be extended to a wider class of string defects: the semi-local strings $^{[7]}$ and the electroweak strings. ${ }^{[8]}$ These recently discovered defects occur in 
theories where the fundamental group of the vacuum manifold is trivial. Thus, they are at best metastable. ${ }^{[9]}$ While Z-flux carrying electroweak strings are unstable in the Weinberg-Salam model, various mechanisms for stabilizing a Z-flux string have been proposed. First, one can add a linear time dependence of the Goldstone boson to obtain a stable spinning vortex solution. ${ }^{[10]}$ Second, fermions that are massive outside the core become massless inside. It is clear that there are superconducting zero modes in the core. These bound states tend to stabilize the non-topological solitons. ${ }^{[11]}$ Another possibility would be to consider extensions of the electroweak model or topological strings carrying Z-flux which are formed in an earlier phase transition. $^{[12]}$

Baryogenesis during the weak phase transition is particularly interesting as it may eventually be experimentally verifiable. Consider the following wild speculative scenario: non-topological electroweak strings are formed at the electroweak phase transition. They are stabilized by some mechanism (either one of the above or a combination or some other means). Baryogenesis occurs inside their cores. Baryogenesis due to electroweak strings in the two-Higgs model has been discussed in the literature. $^{[13]}$ It would be interesting to understand the relevance of our results to baryogenesis in future investigations.

After the completion of an earlier version of this paper, we received a revised manuscript by Davis, Martin and Ganoulis ${ }^{[14]}$ which also discussed electrons scattering off electroweak strings for $0 \leq \sin ^{2} \theta_{w} \leq 1 / 2$ and $k \ll m$ or $k \gg m$. In this paper, we consider the whole parameter space $0 \leq \sin ^{2} \theta_{w} \leq 1$ in the regimé $m \sim k$. In particular, our analysis applies to semi-local strings, which correspond to $\sin ^{2} \theta_{w}=1$.

The plan of this paper is as follows. In section 2, we review the subject of the electroweak strings and describe a simple model of the field configuration that we will be working with. Using a partial wave analysis, the differential cross section for the helicity flip process of electrons for various values of $\theta_{w}$ will be computed in section 3. In particular, we show that the cross section is proportional to $m_{e}^{-1}$ for $0<\sin ^{2} \theta_{w}<1 / 2, \omega \sim k \sim m_{e}$ and $k R \ll 1$. Moreover, the result for the semi-local 
strings can be obtained from that of the electroweak strings by setting $\sin ^{2} \theta_{w}$ to 1 . In our concluding remarks in section 4, we also note that helicity is violated outside the core of an axion string. Thus, it makes no sense to discuss helicity conserving and helicity flip cross sections in this context.

\section{Extended Abelian Higgs Model and Electroweak Strings}

Consider an extension of the Abelian Higgs model with $N=2$ complex scalars $\Phi$ with their overall phase gauged and an $S U(2)$ global symmetry. The most general renormalizable Lagrangian in four dimensions consistent with these symmetries is

$$
\mathcal{L}=\left|D_{\mu} \Phi\right|^{2}-\frac{1}{2} \lambda\left(|\Phi|^{2}-\eta^{2}\right)^{2}-\frac{1}{4} F_{\mu \nu} F^{\mu \nu}
$$

The field $\Phi$ acquires a vacuum expectation value of magnitude $\eta$ and the symmetry is spontaneously broken into a global $U(1)$. It has also been shown that the NielsenOlesen vortex solutions ${ }^{[15]}$ of the Abelian Higgs model (the case with $N=1$ ) carry over to the extended Abelian Higgs model. However, the stability of such vortex solutions becomes a dynamical question and depends on the ratio of the masses of the Higgs and vector particles..$^{[9]}$

Now the extended Abelian Higgs model is precisely the Weinberg-Salam model ${ }^{[16]}$ with the $S U(2)$ charge set equal to zero. By gauging the $S U(2)$ symmetry, one obtains string solutions in the electroweak theory. Such electroweak strings are nontopological and unstable in the minimal electroweak theory. They may, however, be made metastable in some extended models.

Consider an electron moving in the background field of an electroweak string. The relevant part of the Lagrangian is

$$
\mathcal{L}=i \bar{L} \gamma^{\mu} D_{\mu} L+i \bar{e}_{R} \gamma^{\mu} D_{\mu} e_{R}-f_{e}\left(\bar{L} e_{R} \Phi+\Phi^{\dagger} \bar{e}_{R} L\right)
$$

where $\bar{L}=\left(\bar{\nu}, \bar{e}_{L}\right), f_{e}$ is the Yukawa coupling constant, $\Phi$ is the usual Higgs doublet, 
and the covariant derivative has the form

$$
D_{\mu}=\partial_{\mu}+\frac{i \alpha \gamma}{2} Z_{\mu}
$$

where $\gamma=e /\left(\sin \theta_{w} \cos \theta_{w}\right)\left(\theta_{w}\right.$ being the Weinberg angle) and the $Z$-coupling, $\alpha$, is given by ${ }^{[17]}$

$$
\alpha=-2\left(T_{3}-Q \sin ^{2} \theta_{w}\right)
$$

where $T_{3}$ is weak isospin and $Q$ is electric charge. Note that for electrons and down quarks,

$$
\alpha_{L}=\alpha_{R}+1
$$

and there is a marked asymmetry between left and right fields.

For explicit calculations, consider the following simple model ${ }^{[14]}$ of the field configuration.

$$
\begin{aligned}
& \Phi=\left(\begin{array}{ll}
\Phi^{+} & \Phi^{0}
\end{array}\right)=f(r) e^{i \theta}\left(\begin{array}{l}
0 \\
1
\end{array}\right) \\
& Z_{\phi}=-v(r) / r \\
& Z_{r}=W=A=0 \\
& f(r)= \begin{cases}0 & r<R \\
\frac{\eta}{\sqrt{2}} & r>R\end{cases} \\
& v(r)= \begin{cases}0 & r<R \\
\frac{2}{\gamma} & r>R\end{cases}
\end{aligned}
$$

where $Z$ and $W$ are the gauge bosons and $A$ is the photon field. We expect our results to be insensitive to the detail of the core model. A discussion about this issue can be found in Ref. 6 . 


$$
\begin{aligned}
& \text { Writing } e_{L}=\left(\begin{array}{l}
0 \\
\psi
\end{array}\right) \text { and } e_{R}=\left(\begin{array}{l}
\chi \\
0
\end{array}\right) \text {, in the representation } \\
& \gamma^{0}=\left(\begin{array}{ll}
0 & 1 \\
1 & 0
\end{array}\right), \gamma^{i}=\left(\begin{array}{cc}
0 & -\sigma^{j} \\
\sigma^{j} & 0
\end{array}\right), \gamma^{5}=\left(\begin{array}{cc}
1 & 0 \\
0 & -1
\end{array}\right),
\end{aligned}
$$

the Hamiltonian is

$$
H=\left(\begin{array}{cc}
-i \sigma^{j} D_{j}^{R} & f_{e} f e^{-i \theta} \\
f_{e} f e^{i \theta} & i \sigma^{j} D_{j}^{L}
\end{array}\right)
$$

The equations of motion for $\psi$ and $\chi$ are

$$
\begin{aligned}
& \omega \chi+i \sigma^{j} D_{j}^{R} \chi-f_{e} f e^{-i \theta} \psi=0 \\
& \omega \psi-i \sigma^{j} D_{j}^{L} \psi-f_{e} f e^{i \theta} \chi=0 .
\end{aligned}
$$

Note the phase $e^{i \theta}$ and the coupling of $\psi$ to $\chi$ via the mass term. Inside the core, there is no coupling and electron is massless. The helicity operator is given by

$$
\Sigma \cdot \Pi=\left(\begin{array}{cc}
\sigma \cdot \pi_{R} & 0 \\
0 & \sigma \cdot \pi_{L}
\end{array}\right)=\left(\begin{array}{cc}
-i \sigma^{j} D_{j}^{R} & 0 \\
0 & -i \sigma^{j} D_{j}^{L}
\end{array}\right) .
$$

To see that helicity is not conserved, we compute its commutator with the Hamiltonian and find it to be non-zero inside the core. ${ }^{[18]}$

$$
[H, \Sigma \cdot \Pi]=i f_{e}\left(\begin{array}{cc}
0 & \sigma^{j}\left(D_{j} \Phi^{0}\right)^{*} \\
\sigma^{j} D_{j} \Phi^{0} & 0
\end{array}\right)
$$

Note that helicity violating processes can only occur in the string core. They can, however, be enhanced by an amplification of the fermionic wave function at the core radius. In the following section, we perform a detailed calculation of the differential cross section for such scattering processes. 


\section{Scattering Amplitude}

We try the usual partial wave decomposition.

$$
\begin{aligned}
& \chi(r, \theta)=\sum_{l=-\infty}^{\infty}\left(\begin{array}{c}
\chi_{1}^{l}(r) \\
i \chi_{2}^{l}(r) e^{i \theta}
\end{array}\right) e^{i l \theta} \\
& \psi(r, \theta)=\sum_{l=-\infty}^{\infty}\left(\begin{array}{c}
\psi_{1}^{l}(r) \\
i \psi_{2}^{l}(r) e^{i \theta}
\end{array}\right) e^{i(l+1) \theta} .
\end{aligned}
$$

Making use of

$$
\sigma^{j} D_{j}=\left(\begin{array}{cc}
0 & e^{-i \theta}\left(D_{r}-i D_{\theta}\right) \\
e^{i \theta}\left(D_{r}+i D_{\theta}\right) & 0
\end{array}\right),
$$

we substitute (12) into (9) to obtain

$$
\begin{aligned}
& \omega \chi_{2}^{l}+\left(\frac{d}{d r}-\frac{l}{r}+\frac{\alpha_{R} \gamma \nu}{2 r}\right) \chi_{1}^{l}-f_{e} f \psi_{2}^{l}=0 \\
& \omega \chi_{1}^{l}-\left(\frac{d}{d r}+\frac{l+1}{r}-\frac{\alpha_{R} \gamma \nu}{2 r}\right) \chi_{2}^{l}-f_{e} f \psi_{1}^{l}=0 \\
& \omega \psi_{2}^{l}-\left(\frac{d}{d r}-\frac{l+1}{r}+\frac{\alpha_{L} \gamma \nu}{2 r}\right) \psi_{1}^{l}-f_{e} f \chi_{2}^{l}=0 \\
& \omega \psi_{1}^{l}+\left(\frac{d}{d r}+\frac{l+2}{r}-\frac{\alpha_{L} \gamma \nu}{2 r}\right) \psi_{2}^{l}-f_{e} f \chi_{1}^{l}=0 .
\end{aligned}
$$

(a) Internal Solution $(r<R)$

In this region, $f=v=0$, so the equations of motion (14) reduce to

$$
\begin{array}{r}
\omega \chi_{2}^{l}+\left(\frac{d}{d r}-\frac{l}{r}\right) \chi_{1}^{l}=0 \\
\omega \chi_{1}^{l}-\left(\frac{d}{d r}+\frac{l+1}{r}\right) \chi_{2}^{l}=0 \\
\omega \psi_{2}^{l}-\left(\frac{d}{d r}-\frac{l+1}{r}\right) \psi_{1}^{l}=0 \\
\omega \psi_{1}^{l}+\left(\frac{d}{d r}+\frac{l+2}{r}\right) \psi_{2}^{l}=0 .
\end{array}
$$

Thus, $\psi$ and $\chi$ are decoupled from each other in the string core. Combining the first two equations and setting $z=\omega r$, we obtain

$$
\frac{1}{z} \frac{d}{d z}\left(z \frac{d}{d z}\right) \chi_{1}^{l}+\left(\frac{z^{2}-l^{2}}{z^{2}}\right) \chi_{1}^{l}=0 .
$$

This is none other than Bessel's equation of order $l$. By regularity at the origin, the 
solution is

$$
\chi_{1}^{l}=c_{l} J_{l}(\omega r)
$$

This together with the second equation implies

$$
\chi_{2}^{l}=c_{l} J_{l+1}(\omega r)
$$

By a similar argument, $\psi_{1}^{l}$ and $\psi_{2}^{l}$ satisfy Bessel's equations of order $l+1$ and $l+2$ respectively and the internal solution is

$$
\left(\begin{array}{c}
\chi \\
\psi
\end{array}\right)=\sum_{l=-\infty}^{\infty}\left(\begin{array}{c}
c_{l} J_{l}(\omega r) \\
i c_{l} J_{l+1}(\omega r) e^{i \theta} \\
d_{l} J_{l+1}(\omega r) e^{i \theta} \\
i d_{l} J_{l+2}(\omega r) e^{2 i \theta}
\end{array}\right) e^{i l \theta}
$$

\section{(b) External Solution $(r>R)$}

Outside the string core, we decompose our wave functions into eigenfunctions of the helicity operator. i.e.,

$$
\begin{aligned}
& \left(\sigma \cdot \pi_{R}\right) \chi=-i \sigma^{j} D_{j}^{R} \chi= \pm k \chi \\
& \left(\sigma \cdot \pi_{L}\right) \psi=-i \sigma^{j} D_{j}^{L} \psi= \pm k \psi
\end{aligned}
$$

From eqn.(14), this gives

$$
\begin{aligned}
& (\omega \mp k) \chi=f_{e} f \psi=m \psi \\
& (\omega \pm k) \psi=f_{e} f \chi=m \chi
\end{aligned}
$$

Defining

$$
\nu=l-\alpha_{R}
$$

and $z^{\prime}=k r$, eqn.(20) yields

$$
\chi_{2}^{l}=\mp\left(\frac{d}{d z^{\prime}}-\frac{\nu}{z^{\prime}}\right) \chi_{1}^{l},
$$

where $-(+)$ is taken for a positive (negative) helicity state. Thus, the external 
solution is

$$
\left(\begin{array}{c}
\chi \\
\psi
\end{array}\right)=\sum_{l=-\infty}^{\infty}\left(\begin{array}{c}
Z_{\nu}(k r) \\
\pm i Z_{\nu+1}(k r) e^{i \theta} \\
B^{ \pm} Z_{\nu}(k r) e^{i \theta} \\
\pm i B^{ \pm} Z_{\nu+1}(k r) e^{2 i \theta}
\end{array}\right) e^{i l \theta}
$$

In the above, $B^{ \pm}=\frac{m}{\omega \pm k}$, the superscript \pm in $B$ denotes the helicity and \pm in the front of the second and fourth components take the same sign as the helicity for $Z_{\nu}=J_{\nu}, N_{\nu}$ and $H_{\nu}$ and opposite sign for $Z_{\nu}=J_{-\nu}, N_{-\nu}$ and $H_{-\nu}$. Here $N_{\nu}$ and $H_{\nu}$ are Neumann and outgoing Hankel functions respectively. Note that it is $k r$ rather than $\omega r$ which appears in the arguments of our functions because electrons are massive outside the core. Another point to note is that whereas the second and third components of the internal solutions satisfy Bessel's equation of the same order, the corresponding components of the external solutions satisfy Bessel's equations of orders $\nu+1$ and $\nu$ respectively. This relative shift in the order is due to the asymmetry between left and right, i.e., $\alpha_{L}=\alpha_{R}+1$.

\section{(c) Asymptotic Solution}

Consider performing a scattering experiment with an incoming plane wave of positive helicity electrons. Since helicity is violated in the core, the scattered wave consists of both positive and negative helicity components. We find that, as $r \rightarrow \infty$, the external solution takes the form

$$
\sum_{l=-\infty}^{\infty} e^{i l \theta}\left[\left(\begin{array}{c}
(-i)^{l} J_{l} \\
i(-i)^{l} J_{l+1} e^{i \theta} \\
B^{+}(-i)^{l} J_{l} e^{i \theta} \\
i B^{+}(-i)^{l} J_{l+1} e^{2 i \theta}
\end{array}\right)+\frac{f_{l} e^{i k r}}{\sqrt{r}}\left(\begin{array}{c}
1 \\
e^{i \theta} \\
B^{+} e^{i \theta} \\
B^{+} e^{2 i \theta}
\end{array}\right)+\frac{g_{l} e^{i k r}}{\sqrt{r}}\left(\begin{array}{c}
1 \\
-e^{i \theta} \\
B^{-} e^{i \theta} \\
-B^{-} e^{2 i \theta}
\end{array}\right)\right]
$$

It is easy to check that the second and third terms are the positive and negative helicity components of the scattered waves respectively.

We divide the problem of matching the asymptotic wave functions into two cases. 
(i) For $\nu \geq 0$ or $\nu \leq-1$, we take $Z_{\nu}^{1}=J_{\nu}$ and $Z_{\nu}^{2}=N_{\nu}$. The external wave function is therefore

$$
\left(\begin{array}{cccccccc}
\left(a_{l} J_{\nu}\right. & + & b_{l} N_{\nu} & + & A_{l} J_{\nu} & + & \left.B_{l} N_{\nu}\right) e^{i l \theta} \\
i\left(a_{l} J_{\nu+1}\right. & + & b_{l} N_{\nu+1} & - & A_{l} J_{\nu+1} & - & \left.B_{l} N_{\nu+1}\right) e^{i(l+1) \theta} \\
\left(a_{l} B^{+} J_{\nu}\right. & + & b_{l} B^{+} N_{\nu} & + & A_{l} B^{-} J_{\nu} & + & \left.B_{l} B^{-} N_{\nu}\right) e^{i(l+1) \theta} \\
i\left(a_{l} B^{+} J_{\nu+1}\right. & + & b_{l} B^{+} N_{\nu+1} & - & A_{l} B^{-} J_{\nu+1} & - & \left.B_{l} B^{-} N_{\nu+1}\right) e^{i(l+2) \theta}
\end{array}\right)
$$

Making use of the asymptotic large $x$ forms

$$
\begin{aligned}
J_{\mu}(x) & \sim \sqrt{\frac{2}{\pi x}} \cos \left(x-\frac{\mu \pi}{2}-\frac{\pi}{4}\right) \\
N_{\mu}(x) & \sim \sqrt{\frac{2}{\pi x}} \sin \left(x-\frac{\mu \pi}{2}-\frac{\pi}{4}\right),
\end{aligned}
$$

we match coefficients of $e^{i l \theta} \frac{e^{ \pm i k r}}{\sqrt{r}}$ in eqns. (25) and (26) to find

$$
\begin{aligned}
& e^{i \nu \pi / 2} \quad\left(a_{l}+i b_{l}+A_{l}+i B_{l}\right)=1 \\
& e^{-i \nu \pi / 2} \quad\left(a_{l}-i b_{l} \quad+A_{l} \quad-i B_{l}\right)=(-1)^{l}+\left(f_{l}+g_{l}\right) e^{i \pi / 4} \sqrt{2 \pi k} \\
& e^{i(\nu+1) \pi / 2} \quad\left(i a_{l} \quad-b_{l} \quad-i A_{l} \quad+B_{l}\right)=-1 \\
& e^{-i(\nu+1) \pi / 2}\left(i a_{l} \quad+b_{l} \quad-i A_{l} \quad-B_{l}\right)=(-1)^{l}+\left(f_{l}-g_{l}\right) e^{i \pi / 4} \sqrt{2 \pi k},
\end{aligned}
$$

from which we deduce

$$
\begin{aligned}
& A_{l}=-i B_{l} \\
& a_{l}=-i b_{l}+e^{-i \nu \pi / 2} \\
& g_{l}=e^{-i(\pi / 4+\nu \pi / 2)} \sqrt{\frac{1}{2 \pi k}}\left(-2 i B_{l}\right) .
\end{aligned}
$$

(ii) For $-1<\nu<0$, taking $Z_{\nu}^{1}=J_{\nu}$ and $Z_{\nu}^{2}=J_{-\nu}$, the external wave function is 


$$
\left(\begin{array}{cccccccc}
\left(a_{l} J_{\nu}\right. & + & b_{l} J_{-\nu} & + & A_{l} J_{\nu} & + & \left.B_{l} J_{-\nu}\right) e^{i l \theta} \\
i\left(a_{l} J_{\nu+1}\right. & - & b_{l} J_{-\nu-1} & - & A_{l} J_{\nu+1} & + & \left.B_{l} J_{-\nu-1}\right) e^{i(l+1) \theta} \\
\left(a_{l} B^{+} J_{\nu}\right. & + & b_{l} B^{+} J_{-\nu} & + & A_{l} B^{-} J_{\nu} & + & \left.B_{l} B^{-} J_{-\nu}\right) e^{i(l+1) \theta} \\
i\left(a_{l} B^{+} J_{\nu+1}\right. & - & b_{l} B^{+} J_{-\nu-1} & - & A_{l} B^{-} J_{\nu+1} & + & \left.B_{l} B^{-} J_{-\nu-1}\right) e^{i(l+2) \theta}
\end{array}\right)
$$

We proceed as before and find

$$
\begin{aligned}
A_{l} & =-e^{-i \nu \pi} B_{l} \\
a_{l} & =-e^{-i \nu \pi} b_{l}+e^{-i \nu \pi / 2} \\
g_{l} & =\sqrt{\frac{1}{2 \pi k}} e^{-i(\nu \pi / 2+\pi / 4)} 2 i \sin (\nu \pi) B_{l} .
\end{aligned}
$$

Note that

$$
\left.\frac{d \sigma}{d \theta}\right|_{+\rightarrow-}=\sum_{l}\left|g_{l}\right|^{2} .
$$

(d) Matching at $r=R$

We have obtained the solutions inside and outside the core in (a) and (b). Now we match them at $r=R$. Because of the difference in the masses in the two regions and the discontinuous distribution of the string flux, there is a discontinuity in the first derivatives of the wave functions. Nevertheless, the wave functions themselves are continuous at $r=R$. This is the matching condition that we will use ${ }^{[5,6]}$ Once again there are two cases.

(i) $\nu \geq 0$ or $\nu \leq-1$ : Substituting (29) into (26) and matching it with the internal solution in eqn.(19), we obtain

$$
\begin{array}{ccccccc}
-i H_{\nu} b_{l} & - & i H_{\nu} B_{l} & = & J_{l} c_{l} & - & e^{-i \nu \pi / 2} J_{\nu} \\
-i H_{\nu+1} b_{l} & + & i H_{\nu+1} B_{l} & & J_{l+1} c_{l} & - & e^{-i \nu \pi / 2} J_{\nu+1} \\
-i B^{+} H_{\nu} b_{l} & - & i B^{-} H_{\nu} B_{l} & = & J_{l+1} d_{l} & - & e^{-i \nu \pi / 2} B^{+} J_{\nu} \\
-i B^{+} H_{\nu+1} b_{l}+i B^{-} H_{\nu+1} B_{l} & = & J_{l+2} d_{l} & - & e^{-i \nu \pi / 2} B^{+} J_{\nu+1} .
\end{array}
$$

In deriving the above equations, we have used the definition of outgoing Hankel 
function: $H_{\mu}=J_{\mu}+i N_{\mu}$. Solving eqn.(33), we find

$$
B_{l}=\frac{\Delta_{B}}{\Delta}
$$

where

$$
\Delta_{B}=B^{+} e^{-i \nu \pi / 2}\left(\frac{2}{\pi k R}\right)\left(J_{l+1}^{2}-J_{l} J_{l+2}\right)
$$

and

$$
\Delta=\left(B^{-}-B^{+}\right) J_{l+1}\left(J_{l} H_{\nu+1}^{2}-J_{l+2} H_{\nu}^{2}\right)-\left(B^{-}+B^{+}\right)\left(J_{l+1}^{2}-J_{l} J_{l+2}\right) H_{\nu} H_{\nu+1}
$$

where $H_{\nu}$ is outgoing Hankel functions. Use has been made of the Wronskian formula $J_{\nu+1}(x) N_{\nu}(x)-J_{\nu}(x) N_{\nu+1}(x)=\frac{2}{\pi x}$ in the derivation of eqn.(35).

Now we consider the regimé $\omega \sim k \sim m$ and $k R \ll 1$ and perform small $k R$ approximation:

$$
J_{\mu} \sim O\left([k R]^{\mu}\right), \quad H_{\mu}, N_{\mu} \sim O\left([k R]^{-|\mu|}\right) .
$$

It is straightforward, but tedious to show that

$$
B_{l} \propto \begin{cases}(k R)^{2 \nu+2} & \nu \geq 0, l \geq 0 \\ (k R)^{2 \nu} & \nu \geq 0, l<0 \\ (k R)^{-2 \nu-2} & \nu \leq-1, l \geq-1 \\ (k R)^{-2 \nu} & \nu \leq-1, l<-1 .\end{cases}
$$

Note that the cross section may still be logarithmically suppressed when the exponent in the suppression factor appears to be zero.

$$
\text { (ii) }-1<\nu<0
$$


Substituting eqn.(31) into (30) and matching it with the internal solution in eqn.(19), we obtain the following equations.

$$
\begin{aligned}
& P\left(b_{l}+B_{l}\right)=J_{l} c_{l}-e^{-i \nu \pi / 2} J_{\nu} \\
& Q\left(b_{l}-B_{l}\right)=J_{l+1} c_{l}-e^{-i \nu \pi / 2} J_{\nu+1} \\
& P\left(B^{+} b_{l}+B^{-} B_{l}\right)=J_{l+1} d_{l}-e^{-i \nu \pi / 2} B^{+} J_{\nu}, \\
& Q\left(B^{+} b_{l}-B^{-} B_{l}\right)=J_{l+2} d_{l}-e^{-i \nu \pi / 2} B^{+} J_{\nu+1}
\end{aligned}
$$

where $P$ denotes $-e^{-i \nu \pi} J_{\nu}+J_{-\nu}$ and $Q$ denotes $-e^{-i \nu \pi} J_{\nu+1}-J_{-\nu-1}$. Solving (39), we find

$$
B_{l}=\frac{\Delta_{B}^{\prime}}{\Delta^{\prime}}
$$

where

$$
\Delta_{B}^{\prime}=\frac{2 B^{+} \sin (\nu \pi) e^{-i \nu \pi / 2}}{\pi k R}\left(J_{l} J_{l+2}-J_{l+1}^{2}\right)
$$

and

$$
\Delta^{\prime}=\left(B^{-}-B^{+}\right) J_{l+1}\left(J_{l+2} P^{2}-J_{l} Q^{2}\right)+\left(B^{-}+B^{+}\right)\left(J_{l+1}^{2}-J_{l} J_{l+2}\right) P Q
$$

Use has also been made of the Wronskian formula $J_{\nu} J_{-\nu-1}+J_{-\nu} J_{\nu+1}=-\frac{2 \sin (\nu \pi)}{\pi x}$ in deriving eqn.(41).

We consider the regimé $\omega \sim k \sim m$ and $k R \ll 1$. A straightforward calculation shows that

$$
B_{l} \propto \begin{cases}(k R)^{2 \nu+2} & l \geq 0 \\ (k R)^{-2 \nu} & l \leq-2 \\ 1 & l=-1\end{cases}
$$

We see immediately that when the last case occurs, the mode $l=-1(-1<\nu<0)$ dominates the contribution from all other modes, and is of order 1 . In that case, the helicity flip process is maximally enhanced with a cross section of order $1 / m$, where $m$ is the mass of the incoming particle. Recalling that $\nu=l-\alpha_{R}$, we see that this occurs precisely when $-1<\alpha_{R}<0$ (and thus $0<\alpha_{L}<1$.) For electrons $\alpha_{R}=-2 \sin ^{2} \theta_{w}$ and the condition reduces to $0<\sin ^{2} \theta_{w}<1 / 2$. 
Let us consider the changes in the helicity flip cross section as $\sin ^{2} \theta_{w}$ increases from 0 to 1 in the regimé $\omega \sim k \sim m$ and $k R \ll 1$.

(1) $\sin ^{2} \theta_{w}=0\left(\alpha_{R}=0\right)$

(2) $0<\sin ^{2} \theta_{w}<\frac{1}{2}\left(-1<\alpha_{R}<0\right)$

(3) $\sin ^{2} \theta_{w}=\frac{1}{2}\left(\alpha_{R}=-1\right)$

(4) $\frac{1}{2}<\sin ^{2} \theta_{w}<\frac{3}{4}\left(-1.5<\alpha_{R}<-1\right)$

(5) $\sin ^{2} \theta_{w}=\frac{3}{4}\left(\alpha_{R}=-1.5\right)$

(6) $\frac{3}{4}<\sin ^{2} \theta_{w}<1\left(-2<\alpha_{R}<-1.5\right)$

(7) $\sin ^{2} \theta_{w}=1\left(\alpha_{R}=-2\right)$

Before embarking on a discussion about the various cases for electrons, we would like to remark that the results for down quarks are similar. It is still true that $\alpha_{L}=\alpha_{R}+1$. The only difference is that $\alpha_{R}=-\frac{2}{3} \sin ^{2} \theta_{w}$ for d quarks. Therefore, there are just two cases. If $\sin ^{2} \theta_{w}=0$, the result is the same as in case (1) for electrons and the helicity flip scattering has, up to normalization, an Everett's cross section. (Cf. case (1) below.) If $0<\sin ^{2} \theta_{w} \leq 1$, there is a maximal enhancement and the cross section per unit length $\sim 1 / m_{d}$. (Cf. case (2) below.) Now we turn to electrons.

(1) For $\sin ^{2} \theta_{w}=0\left(\alpha_{R}=0\right)$, the $l=\nu=-1$ mode dominates and from eqns. (29), (32) and (34)-(36), the differential cross section per unit length

$$
\frac{d \sigma}{d \theta} \sim \frac{1}{k \ln ^{2}(k R)}
$$

which is, up to normalization, the cross section obtained by Everett ${ }^{[19]}$ for the scattering of scalar particles off cosmic strings with integral magnetic fluxes.

(2) For $0<\sin ^{2} \theta_{w}<\frac{1}{2}\left(-1<\alpha_{R}<0\right)$, from eqns. (31), (32) and (43), the 
electron helicity flip process has a differential cross section

$$
\frac{d \sigma}{d \theta}=O\left(m_{e}^{-1}\right)
$$

which is dominated by the mode $-1<\nu<0(l=-1)$ and is thus independent of angle. In this case, the helicity flip process remains unsuppressed as $R \rightarrow \infty$ with $k$ held fixed. Note that this maximal amplification occurs for a continuous range of values of the parameter $\sin ^{2} \theta_{w}$. This is in contrast with an analogous calculation on baryon number violating processes due to cosmic strings which exhibit unsuppressed cross section for only discrete values of fluxes. ${ }^{[5,6]}$

(3) For $\sin ^{2} \theta_{w}=\frac{1}{2}\left(\alpha_{R}=-1\right)$, the $l=-1, \nu=0$ mode swamps contributions from all other modes. Eqns. (29), (32) and (34)-(36) together implies that the cross section is of the same order as in case (1).

(4) For $\frac{1}{2}<\sin ^{2} \theta_{w}<\frac{3}{4}\left(-1.5<\alpha_{R}<-1\right)$, the dominant mode is $0<\nu<0.5$ $(l=-1)$. From eqns. (29), (32) and (38), the differential cross section is given by

$$
\frac{d \sigma}{d \theta} \sim k^{-1}(k R)^{4 \nu}=k^{-1}(k R)^{4\left(2 \sin ^{2} \theta_{w}-1\right)}
$$

(5) For $\sin ^{2} \theta_{w}=\frac{3}{4}\left(\alpha_{R}=-1.5\right)$, the two modes $l=-1$ and -2 give comparable contributions and we obtain from eqns. (29), (31), (32), (38) and (43) that

$$
\frac{d \sigma}{d \theta} \sim \frac{1}{k(k R)^{2}}\left|1+C e^{i \theta}\right|^{2}
$$

(6) For $\frac{3}{4}<\sin ^{2} \theta_{w}<1\left(-2<\alpha_{R}<-1.5\right)$, we need to consider the contribution from the $l=-2$ mode only and obtain from eqns. (31), (32) and (43) that

$$
\frac{d \sigma}{d \theta} \sim k^{-1}(k R)^{8\left(1-\sin ^{2} \theta_{w}\right)}
$$

(7) For $\sin ^{2} \theta_{w}=1\left(\alpha_{R}=-2\right)$, the $l=-2, \nu=0$ mode will dominate and the 
differential cross section can be deduced from eqns. (29), (32) and (34)-(36):

$$
\frac{d \sigma}{d \theta} \sim \frac{1}{k \ln ^{4}(k R)}
$$

Note that the exponent of the logarithmic term is four, whereas in cases (1) and (3) it is two. We note on passing that case (7) corresponds to semi-local strings, where the $S U(2)$ gauge charge is set to zero.

The most prominent feature of our result is the presence of a plateau: For $\sin ^{2} \theta_{w}$ between 0 and $1 / 2$, we have maximal enhancement. Is there any heuristic way of understanding its origin? In Ref. 6, the cross section for a baryon number violating process was derived using first order perturbation theory in quantum field theories. The transition matrix element between an initial state $|\psi\rangle$ and a final state $\left|\psi^{\prime}\right\rangle$ is given by $\mathcal{A}=\left\langle\psi^{\prime}\left|\int d^{4} x \mathcal{L}_{I}(x)\right| \psi\right\rangle$. The computation is divided into two steps. Firstly, we evaluate $\mathcal{A}$ using free fermion spinors, resulting in the "geometric" cross section. In the second step, we solve the Dirac equation with the appropriate boundary conditions to determine the amplitude of the spinor at the core radius $\mathrm{R}$ and define the amplification factor $A$ as the ratio of the amplitude of the actual spinor to that of a free spinor. Since the cross section is proportional to $\mathcal{A}^{2}$ and since $\mathcal{A}$ involves two spinors, the catalysis cross section is enhanced by a factor $A^{4}$ over the geometric cross section. Now we attempt a similar discussion for helicity flip due to electroweak strings. The difficulty of such an approach lies in the decomposition of the Hamiltonian into helicity conserving and helicity violating parts. The point is that the Yukawa coupling between the Higgs field and electrons, apart from giving rise to helicity violation, also makes electrons massive and it seems difficult to separate these two effects. The simplest way out is to consider another object instead, namely the commutator of the helicity operator with the Hamiltonian. This object is clearly proportional to the transition matrix element that we are interested in. From eqn.(11) we see that this commutator couples the first component of a spinor with the fourth

\footnotetext{
* We thank Ming Lu and Piljin Yi for helpful discussions about this.
} 
and the second with the third, etc. In the same spirit as in Ref.6, we compute the coefficients $a_{l}, b_{l}, A_{l}$ and $B_{l}$ of our wave function. For the region $0<\sin ^{2} \theta_{w}<1 / 2$, we find that the mode $-1<\nu<0$ have all coefficients of order unity. It is a simple matter to check that, at the core radius, the first and third components of the initial (+ helicity) wave function are enhanced by a factor $(k R)^{\nu}$ and the second and fourth by $(k R)^{-\nu-1}$. A similar analysis holds for the final ( - helicity) state. Now we have the interesting result that all components are amplified by factors very sensitive to the fractional flux of the string, but the first and third components have a different amplification factor from that of the second and fourth such that when we take the product of the amplification factors, we get an enhancement factor of $(k R)^{-1}$, which is independent of $\alpha_{R}$. This is the origin of the plateau.

\section{Concluding Remarks}

(1) We work in the regimé $\omega \sim k \sim m_{e}$ and $k R \ll 1$. Using a partial wave decomposition, we show that for $0<\sin ^{2} \theta_{w}<1 / 2$, electrons scattering off an electroweak string have a helicity flip cross section (per unit length) of order $m_{e}^{-1}$. This huge cross section is due to an amplification of the fermionic wave function at the core. Within this region of the parameter space, it is found that one partial wave (the mode $-1<\nu<0$ ) dominates the contributions from all other modes, giving an angle independent differential cross section (per unit length) $\propto m_{e}^{-1}$.

(2) Whereas baryon number violating processes are maximally enhanced only for discrete values of the fractional flux, our results show that electroweak strings have maximal amplified helicity flip scattering amplitude for a continuous region of the parameter space $0<\sin ^{2} \theta_{w}<1 / 2$. This is due to the asymmetry between left and right and a subsequent delicate cancellation of the dependence of the overall amplification factor on the Weinberg angle: We consider the commutator between the helicity operator and the Hamiltonian. This commutator gives a coupling between the first and fourth components as well as between the second and third components of the spinor. By computing the coefficients of the wave function, one observes that 
the first and third components are amplified at a factor which is very sensitive to the string flux and is different from the amplification factor for the second and fourth components. However, when we take the product of these two amplification factors to obtain the total amplification factor, we find it to be independent of the string flux, thus resulting in maximal enhancement for a continuous region of the parameter space.

(3) The case $0 \leq \sin ^{2} \theta_{w} \leq 1 / 2$ has been discussed in a revised version of Ref. 14. Can one rederive their results from our discussion? The answer is affirmative. For $0<\sin ^{2} \theta_{w}<1 / 2$ and $\omega R, k R \ll 1$, one can deduce from eqns. (31), (32) and (40)-(42) that

$$
\frac{d \sigma}{d \theta} \approx \frac{2}{\pi k}\left(\frac{m^{2}}{\omega(\omega+k)}\right)^{2} \sin ^{2} \pi \alpha_{R}
$$

(Here we have reinstated the mild $\sin ^{2} \pi \alpha_{R}$ dependence that we have ignored in section 3.) In the limit $k \ll m$, this gives

$$
\frac{d \sigma}{d \theta} \approx \frac{2}{\pi k} \sin ^{2} \pi \alpha_{R}
$$

In the opposite limit $k \gg m$,

$$
\frac{d \sigma}{d \theta} \approx \frac{1}{2 \pi k}\left(\frac{m}{k}\right)^{4} \sin ^{2} \pi \alpha_{R}
$$

We note that the vanishing of the differential cross section in the massless limit can be deduced directly from eqn.(11). Similarly, for $\sin ^{2} \theta_{w}=0$ or $1 / 2$, one deduces from eqns.(29), (32) and (34)-(36) that

$$
\frac{d \sigma}{d \theta} \approx \frac{\pi}{8 k}\left(\frac{m^{2}}{\omega(\omega+k)}\right)^{2} \frac{1}{\ln ^{2} k R}
$$

For $k \ll m$, this gives

$$
\frac{d \sigma}{d \theta} \approx \frac{\pi}{8 k} \frac{1}{\ln ^{2} k R}
$$


For $k \gg m$, this gives

$$
\frac{d \sigma}{d \theta} \approx \frac{\pi}{32 k}\left(\frac{m}{k}\right)^{4} \frac{1}{\ln ^{2} k R}
$$

These results are in good agreement with those of Ref. 14. (The authors gave zero as the final answer to the case $k \gg m$, but it is clear from their arguments that they had neglected $(k / m)^{4}$ terms.)

(4) The cross section for other values of $\theta_{w}$ are also computed. In particular, a semi-local string is none other than an electroweak string with $\sin ^{2} \theta_{w}=1$.

(5) For axion strings, the covariant derivatives in eqn.(11) should be replaced by partial derivatives. Therefore, helicity is violated outside the core and it makes no sense to talk about helicity conserving and helicity flip cross sections in this context.

(6) It would be interesting to investigate the relevance of this work to electroweak baryogenesis. Such scenarios are highly testible.

We gratefully acknowledge useful discussions with Ming Lu, Piljin Yi, and particularly John Preskill. We also thank A. P. Martin for sending us a revised version of Ref. 14 and pointing out some errors in an earlier version of this manuscript.

\section{REFERENCES}

1. C. Callan, Phys. Rev. D25, 2141 (1982); Phys. Rev. D26, 2058 (1982); Nucl. Phys. B212, 391 (1983).

2. V. Rubakov, JETP Lett. 33, 644 (1981); Nucl. Phys. B203, 311 (1982).

3. F. Wilczek, Phys. Rev. Lett. 48, 1146 (1982).

4. R. H. Brandenberger, A.-C. Davis, and A. Matheson, Nucl. Phys. B307, 909 (1988); R. H. Brandenburger and L. Perivolaropoulos, Phys. Lett. B208, 396 (1988).

5. M. G. Alford, J. March-Russell, and F. Wilczek, Nucl. Phys. B328, 140 (1989). 
6. W. B. Perkins, L. Perivolaropoulos, A.-C. Davis, R. H. Brandenberger, and A. Matheson, Nucl. Phys. B353, 237 (1991).

7. T. Vachaspati and A. Achúcarro, Phys. Rev. D 44, 3067 (1991).

8. T. Vachaspati, Phys. Rev. Lett. 68, 1977 (1992).

9. M. Hindmarsh, Phys. Rev. Lett. 68, 1263 (1992); M. James, L. Perivolaropoulos, and T. Vachaspati, Phys. Rev. D 46, 5232 (1992); Nucl. Phys. B 395, 534 (1993).

10. L. Perivolaropoulos, hep-ph/9403298.

11. T. Vachaspati and R. Watkins, Phys. Lett. B318, 163 (1993); T. D. Lee, Phys. Rep. 23C, 254 (1976).

12. G. Dvali and G. Senjanović, hep-ph/9403277; R. Brandenburger, A.-C. Davis, and M. Trodden, hep-ph/9403215.

13. See M. Barriola, hep-ph/9403323 and references therein.

14. A.-C. Davis, A. P. Martin, and N. Ganoulis, Cambridge Preprint DAMTP 93-46.

15. H. B. Nielsen and P. Olesen, Nucl. Phys. B 61, 45 (1973).

16. S. Weinberg, Phys. Rev. Lett. 19, 1264 (1967); A. Salam, in Proceedings of the Eighth Nobel Symposium, ed. N. Svartholm (Almqvist and Wiksell, Stockholm, 1968).

17. J. Preskill, Phys. Rev. D46, 4218 (1992).

18. N. Ganoulis, Phys. Lett. B298, 63 (1993).

19. A. E. Everett, Phys. Rev. D 24, 858 (1981). 